\title{
Arsenate reduction and expression of multiple chromosomal ars operons in Geobacillus kaustophilus A1

\begin{abstract}
Correspondence
Elisabetta Bini

bini@aesop.rutgers.edu
\end{abstract} \\ Received 30 January 2011 \\ Revised 6 April 2011 \\ Accepted 14 April 2011 \author{
and Elisabetta Bini ${ }^{1}$ \\ ${ }^{1}$ Department of Biochemistry and Microbiology, Rutgers, The State University of New Jersey, \\ 76 Lipman Drive, New Brunswick, NJ 08901-0231, USA \\ ${ }^{2}$ Department of Environmental Sciences, Rutgers, The State University of New Jersey, \\ 14 College Farm Road, New Brunswick, NJ 08901-8551, USA
} \\ Mariola Cuebas, ${ }^{1} \ddagger$ Aramis Villafane, ${ }^{1} \dagger$ Michelle McBride, ${ }^{1}$ Nathan $\mathrm{Yee}^{2}$

\begin{abstract}
Geobacillus kaustophilus strain A1 was previously isolated from a geothermal environment for its ability to grow in the presence of high arsenate levels. In this study, the molecular mechanisms of arsenate resistance of the strain were investigated. $\mathrm{As}(\mathrm{V})$ was reduced to $\mathrm{As}(\mathrm{III})$, as shown by HPLC analysis. Consistent with the observation that the micro-organism is not capable of anaerobic growth, no respiratory arsenate reductases were identified. Using specific PCR primers based on the genome sequence of G. kaustophilus HTA426, three unlinked genes encoding detoxifying arsenate reductases were detected in strain $\mathrm{A} 1$. These genes were designated ars $\mathrm{C} 1$, ars $\mathrm{C} 2$ and ars $\mathrm{C} 3$. While ars $\mathrm{C} 3$ is a monocistronic locus, sequencing of the regions flanking ars $\mathrm{C} 1$ and ars $\mathrm{C} 2$ revealed the presence of additional genes encoding a putative arsenite transporter and an ArsR-like regulator upstream of each arsenate reductase, indicating the presence of sequences with putative roles in $\mathrm{As}(\mathrm{V})$ reduction, $\mathrm{As}(\mathrm{III})$ export and arsenic-responsive regulation. $\mathrm{RT}$-PCR demonstrated that both sets of genes were co-transcribed. Furthermore, ars 1 and arsC2, monitored by quantitative real-time RT-PCR, were upregulated in response to $\mathrm{As}(\mathrm{V})$, while ars $\mathrm{C} 3$ was constitutively expressed at a low level. A mechanism for regulation of $\mathrm{As}(\mathrm{V})$ detoxification by Geobacillus that is both consistent with our findings and relevant to the biogeochemical cycle of arsenic and its mobility in the environment is proposed.
\end{abstract}

\section{INTRODUCTION}

Micro-organisms have an important impact on the biogeochemical transformations of arsenic, and their activities affect the mobility and toxicity of this element. Elevated amounts of arsenic can occur, especially in geothermal environments (Stauffer \& Thompson, 1984). Thus, thermophilic micro-organisms such as Geobacillus species that thrive in geothermal soils and sediments are of particular interest for studying the mechanisms for the detoxification of arsenic compounds.

‡Deceased

tPresent address: Department of Biology, University of Puerto Rico, San Juan, Puerto Rico.

Abbreviations: LMWP family, low-molecular-weight phosphatase family; qRT-PCR, quantitative real-time PCR.

The GenBank/EMBL/DDBJ accession numbers for the sequences of G. kaustophilus A1 are FJ602546-FJ602548.

Two supplementary figures and a supplementary table are available with the online version of this paper.
Genes for arsenic detoxification were first discovered and characterized in Gram-negative bacteria (Chen et al., 1986; Rosen, 1999). These genes are often plasmid-encoded and are widespread in prokaryotes (Bruhn et al., 1996; Ji \& Silver, 1992b; Mukhopadhyay \& Rosen, 2002; Mukhopadhyay et al., 2002; Oden et al., 1994; Rosenstein et al., 1992). In thoroughly studied systems, the ars operon has been reported to contain the five genes arsRDABC, as in Escherichia coli (Cai \& DuBow, 1996; Rosen et al., 1992), or at least the three genes arsRBC, as in Staphylococcus aureus (Silver et al., 1993). ArsA and ArsB are components of an arsenite-transporting ATPase, where ArsA is the ATPase and ArsB is the transmembrane component of the complex. In micro-organisms in which ArsA is absent, ArsB acts as a single-component transporter. Both ArsA and ArsB may confer resistance to arsenite and antimonite (Rosen, 1999). In its dimeric form, ArsR is an arsenite-responsive repressor that binds to the ars promoter (Xu \& Rosen, 1997). ArsC is the arsenate reductase that converts arsenate to arsenite, conferring resistance to arsenate (Martin et al., 2001). More recently, new arrangements of arsenic-resistance genes have been discovered in a 
number of micro-organisms, including Bacteria (AchourRokbani et al., 2010; Branco et al., 2008; Li \& Krumholz, 2007; Murphy \& Saltikov, 2009; Ordóñez et al., 2005) and Archaea (Gihring et al., 2003; Wang et al., 2004).

Arsenic resistance and its genetic determinants have not been investigated before in Geobacillus. Among the closest relatives of Geobacillus, arsenic-resistant members of the genus Bacillus have been described that are capable of tolerating arsenate levels up to $20 \mathrm{mM}$ (Bacillus indicus, Bacillus arsenicus) (Shivaji et al., 2005), but the genetic determinants and molecular bases of arsenic resistance in these species are unknown. Furthermore, a number of Bacillus species, including Bacillus arsenicoselenatis, Bacillus selenitireducens (Switzer Blum et al., 1998) and strains isolated from arsenic-rich environments (Fisher \& Hollibaugh, 2008; Santini et al., 2004), have been reported to use $\mathrm{As}(\mathrm{V})$ as the terminal electron acceptor. Such species rely on respiratory arsenate reductases, a class of enzymes unrelated to detoxifying arsenate reductases.

Here we report the molecular characterization of arsenic resistance in Geobacillus kaustophilus strain A1, previously isolated from a geothermal soil on the Metallifere Hills (Italy) and able to tolerate up to $80 \mathrm{mM} \mathrm{As}(\mathrm{V})$ (Cuebas et al., 2011). We have demostrated arsenate reduction, identified the genes responsible for arsenic resistance, and monitored their expression in strain A1. Two arsenate reductases are induced in the presence of $\operatorname{As}(\mathrm{V})$, and a third one is constitutively expressed. Based on these observations, a role for these genes and their regulation in arsenic detoxification is proposed.

\section{METHODS}

Culture conditions. Cells were cultured at $55{ }^{\circ} \mathrm{C}$ in Luria-Bertani (LB) broth. Batch cultures were inoculated to obtain a density corresponding to $\mathrm{OD}_{540} \sim 0.025$, with aliquots withdrawn from midexponential phase $\left(\mathrm{OD}_{540} 0.3-0.6\right)$ cultures. For quantification of gene expression, cell cultures were treated with $5 \mathrm{mM}$ sodium arsenate during the exponential phase of growth. Growth was monitored at $540 \mathrm{~nm}$ on a Beckman DU-520 spectrophotometer (Beckman Coulter).

Analysis of As species. To monitor the concentration of $\mathrm{As}(\mathrm{V})$ and As(III) during growth, cells were cultured on minimal medium (MM) containing $10 \mathrm{mM}$ ammonium sulfate, $2 \mathrm{mM}$ potassium phosphate, $2 \mathrm{mM}$ magnesium phosphate, $0.5 \mathrm{mM}$ calcium chloride, $0.1 \mathrm{mM}$ iron (III) chloride, $0.01 \mathrm{mM}$ sodium tetraborate, $9 \mathrm{mM}$ manganese (II) chloride, $0.75 \mu \mathrm{M}$ zinc sulfate, $0.37 \mu \mathrm{M}$ copper chloride, $0.125 \mu \mathrm{M}$ sodium molybdate, $0.185 \mu \mathrm{M}$ vanadyl (IV) sulfate and $0.065 \mu \mathrm{M}$ cobalt sulfate, $\mathrm{pH} 6.5$, supplemented with $0.2 \%$ sucrose as the carbon and energy source. The medium was amended with $20 \mathrm{mM} \mathrm{As}(\mathrm{V})$, and aliquots were collected every $2 \mathrm{~h}$ and filtered using a $0.45 \mu \mathrm{m}$ pore-size nylon disposable filter to remove cells. The filtrate was then analysed by HPLC. Calibration standards were prepared using matrix-matching background solutions containing MM and known amounts of arsenate and arsenite. MM containing $20 \mathrm{mM} \mathrm{As}(\mathrm{V})$ without cells was used as a control. Measurements of cellular arsenic uptake were conducted by growing the cultures on $\mathrm{MM}$ with 5 or $20 \mathrm{mM}$ As(V) and collecting samples during the exponential phase of growth. Cells were centrifuged, washed with $\mathrm{MM}$ and digested in ultrapure concentrated nitric acid for $20 \mathrm{~h}$ at room temperature. The total arsenic content was determined by inductively coupled plasma optical emission spectroscopy (ICP-OES). Cells grown in the absence of As(V) were used as a control. The concentrations of $\mathrm{As}(\mathrm{V})$ and $\mathrm{As}(\mathrm{III})$ were determined by HPLC. A high-performance liquid chromatograph (Shimadzu) was used to measure arsenite [As(III)] and arsenate $[\mathrm{As}(\mathrm{V})]$ concentrations. The instrument had a Hamilton PRP-X100 anion-exchange column and a UV absorbance detector set at $195 \mathrm{~nm}$.

DNA isolation and sequence analysis. Genomic DNA was isolated as previously described (Cuebas et al., 2011). Briefly, cell pellets were washed and resuspended in $10 \mathrm{mM}$ Tris/ $\mathrm{HCl}, \mathrm{pH} 8.0$, containing $20 \%(\mathrm{v} / \mathrm{v})$ sucrose, and incubated with $2.5 \mathrm{mg}$ lysozyme $\mathrm{ml}^{-1}$ for $45 \mathrm{~min}$ at $37{ }^{\circ} \mathrm{C}$, followed by a second incubation for $2 \mathrm{~h}$ at $55{ }^{\circ} \mathrm{C}$ with Tris-EDTA buffer, $10 \%$ SDS and proteinase K. Finally, the DNA was extracted with phenol/chloroform and treated with RNase A. Segments of ars operons were amplified using pairs of specific primers (Supplementary Table S1) in $25 \mu$ l PCRs assembled with iProof DNA polymerase (Bio-Rad). The strategy to design the primers is described in Results. PCR products were sequenced by GENEWIZ, and the sequences obtained in this way were assembled with BioEdit. For maximum-parsimony analysis, ArsC sequences from G. kaustophilus HTA426 (GK) and strain A1 were aligned to characterized and putative homologues of detoxifying arsenate reductases using CLUSTAL w (Larkin et al., 2007). The alignment was resampled 100 times with Bootstrap, and analysed with Protpars, and a consensus tree was derived applying the program CONSENSE, included within the PHYLIP package (Felsenstein, 2004).

RNA isolation and expression analyses. Total RNA was isolated from exponentially growing cultures $\left(\mathrm{OD}_{540} 0.3-0.6\right)$. Cells were mixed with two volumes of RNAprotect (Qiagen) and centrifuged at 10000 r.p.m. for $1 \mathrm{~min}$ in an Eppendorf microcentrifuge (Brinkmann Instruments, Inc.). RNA was extracted from the cell pellets using the RNeasy Mini kit (Qiagen) and treated with DNase I (Ambion), as recommended by the manufacturers. Absence of contaminating DNA was confirmed by PCR using primers for amplification of the 16S rRNA gene. The quantity and quality of the RNA obtained were evaluated both spectrophotometrically on a NanoDrop ND-1000 spectrophotometer (NanoDrop) and by agarose gel electrophoresis (Sambrook et al., 1989). For RT-PCR analysis, total RNA $(0.5 \mu \mathrm{g})$ was reverse-transcribed, and the cDNA thus obtained was amplified by PCR in $25 \mu$ reactions using the Enhanced Avian HS RT-PCR kit (Sigma-Aldrich). The amplification products were separated on a $1.2 \%$ agarose gel by electrophoresis, and the gel images were acquired using a Gel Logic 440 Imaging system (Eastman Kodak). Furthermore, absolute quantification of specific transcripts was performed by quantitative realtime PCR (qRT-PCR) using the iScript One-Step RT-PCR kit with SYBR Green (Bio-Rad) and the real-time detection system iCycler iQ (Bio-Rad). Reactions, in triplicate, were carried out according to the protocol of the manufacturer, and used $0.2 \mu \mathrm{g}$ total RNA in a $25 \mu \mathrm{l}$ reaction. Two negative controls were included, one without reverse transcriptase and one omitting the template. Primers were designed using the OligoPerfect Designer software (Invitrogen) to have a composition that was suitable for use in both RT-PCR and qRT-PCR (Supplementary Table S1). Standard curves were constructed for each of the three ars $C$ genes using dilutions (range of standards: $10^{4}-10^{9}$ copies $\mu \mathrm{l}^{-1}$ ) of the corresponding PCR amplicons (Chini et al., 2007; Mensink et al., 1998). In parallel, the level of the $r p o B$ transcript, detected using the primer pair QrpoB-F and QrpoB-R (Supplementary Table S1), was monitored as the internal standard to ensure that equal amounts of RNA were used in each reaction. 


\section{RESULTS}

\section{G. kaustophilus strain A1 reduces arsenate}

To determine whether strain Al assimilated arsenate during growth, cells were digested in nitric acid and the total arsenic accumulated was measured. The total amount of arsenic detected in cells grown in the presence of $20 \mathrm{mM}$ arsenate (equivalent to $1.5 \mathrm{~g}$ arsenic $1^{-1}$ ) was approximately $4.4 \mathrm{~g} \mathrm{l}^{-1}$, which is three orders of magnitude lower than the external concentration. Thus, intracellular accumulation of arsenate was ruled out. Because arsenate is known to enter the cell through phosphate channels, we hypothesized that a mechanism for maintaining a low cytoplasmic $\mathrm{As}(\mathrm{V})$ was in place. Therefore, to determine whether strain $\mathrm{Al}$ had the ability to reduce $\mathrm{As}(\mathrm{V})$ to $\mathrm{As}(\mathrm{III})$ and transport it outside the cell, we monitored both oxyanions in a time-course experiment. The results indicated that $\mathrm{A} 1$ is capable of reducing arsenate to arsenite during growth (Fig. 1). Degenerate primers targeted to the arrA genes (Pérez-Jiménez et al., 2005) did not yield amplification (data not shown), and BLASTP similarity searches of the GK genome did not reveal any homologues of ArrA or ArrA-related sequences, e.g. ArxA (Zargar et al., 2010), indicating that respiratory arsenate reductases are absent in strain A1 or highly divergent from known proteins. From this observation, in combination with the fact that Geobacillus A1 requires oxygen for growth (Cuebas et al., 2011), it was postulated that the reduction of $\mathrm{As}(\mathrm{V})$ is most likely a process of detoxification.

\section{Identification of the genetic determinants of $\mathrm{As}(\mathrm{V})$ resistance}

Arsenic resistance is commonly mediated by detoxifying arsenate reductases. Since strain A1 is resistant to high $\mathrm{As}(\mathrm{V})$ and able to reduce it to As(III), we tested by PCR amplification whether the strain possesses an arsenate reductase. GK encodes two clusters of ars genes and a

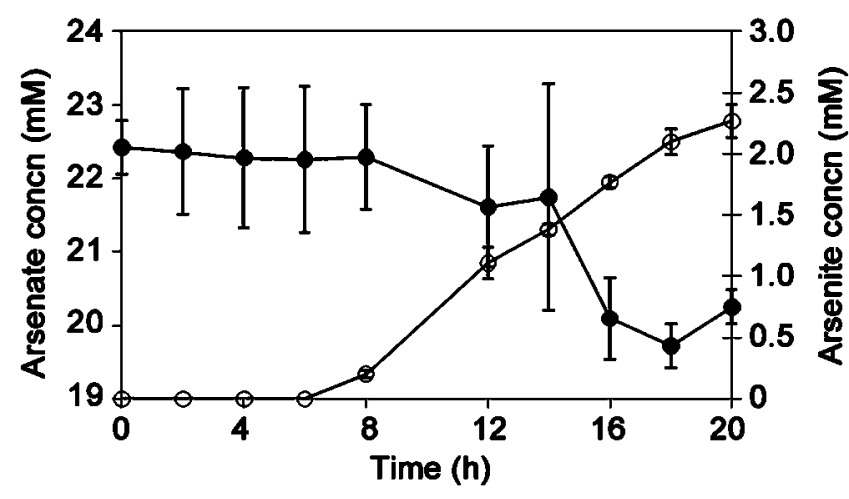

Fig. 1. Time-course analysis of arsenate transformation in MM. Arsenic species were measured by HPLC. ๑, Arsenate concentration; $\bigcirc$, arsenite concentration. Experiments were performed in triplicate; error bars, SD. monocistronic detoxifying arsenate reductase. It cannot be excluded that strain Al possesses additional factors that contribute to arsenic resistance. However, given that both GK and A1 show the same level of arsenate resistance (Cuebas et al., 2011), and no obvious additional sequences with roles in arsenic detoxification have been identified in the GK genome, we focused on the analysis of the putative ars sequences. Therefore, primers for the amplification of the two ars clusters and the monocistronic reductase were designed on the basis of the published GK genome (Takami et al., 2004). To ensure reductase-specificity of the primers, the genome segments spanning from sequence GK3224 to GK3222, and from GK0587 to GK0589, were aligned using CLUSTAL W, and the most dissimilar regions between homologous sequences were selected as targets for the design of PCR primers. First, each pair of primers was tested on genomic DNA isolated from GK (Fig. 2a for location, Supplementary Table S1 for primer sequences). All pairs of primers produced amplicons and their specificity was confirmed by sequencing.

Then, the same pairs of primers were used for PCR amplifications using DNA isolated from strain A1 as template. In this way we obtained the sequences of the segments ars 1 and ars2, each of which included a partial $\operatorname{ars} R$ gene, a full $\operatorname{ars} C$ and a partial $\operatorname{ars} B$ gene (Fig. 2a). The nucleotide sequence of each fragment displayed a high degree of conservation with respect to homologous sequences in GK. In fact, the partial sequences of ars1 and ars 2 were 91 and $98 \%$ identical to the corresponding sequences of GK, respectively. We also obtained the partial nucleotide sequence of strain A1 arsC3 (362 bp), which was $100 \%$ identical to its GK homologue. At the protein level, the ArsB1 transporters and ArsC1 reductases of GK and strain A1 were 94 and $95 \%$ identical (97 and $98 \%$ similar), respectively, while the ArsB2 pumps and $\mathrm{ArsC} 2$ reductases were both $99 \%$ identical ( 99 and $100 \%$ similar, respectively) to their GK counterparts.

\section{Sequence analysis of arsenate reductases}

Two clusters of genes related to arsenate detoxification are found at three distant locations within the GK chromosome, and each includes a transcriptional regulator, an arsenite pump and an arsenate reductase. Both sets of genes display a high level of similarity to one another, suggesting that they might be the result of a duplication event. The proteins encoded by GK3222 (ArsC1, 139 aa) and GK0589 (ArsC2, 140 aa) are $>91 \%$ identical (99\% similar) to each other, and fall in a well-defined clade that comprises arsenate reductases belonging to the low-molecular-weight phosphatase (LMWP) family (Fig. 3). The representative member of this type of reductase was originally characterized in S. aureus (Ji \& Silver, 1992a). This family of proteins is characterized by the presence of the tyrosine-specific protein phosphatase (PTP) signature motif $\mathrm{CX}_{5} \mathrm{R}$, also known as a P-loop (Jackson \& Denu, 2001), located in the $\mathrm{N}$-terminal region and containing the active site. Arsenate 
(a)
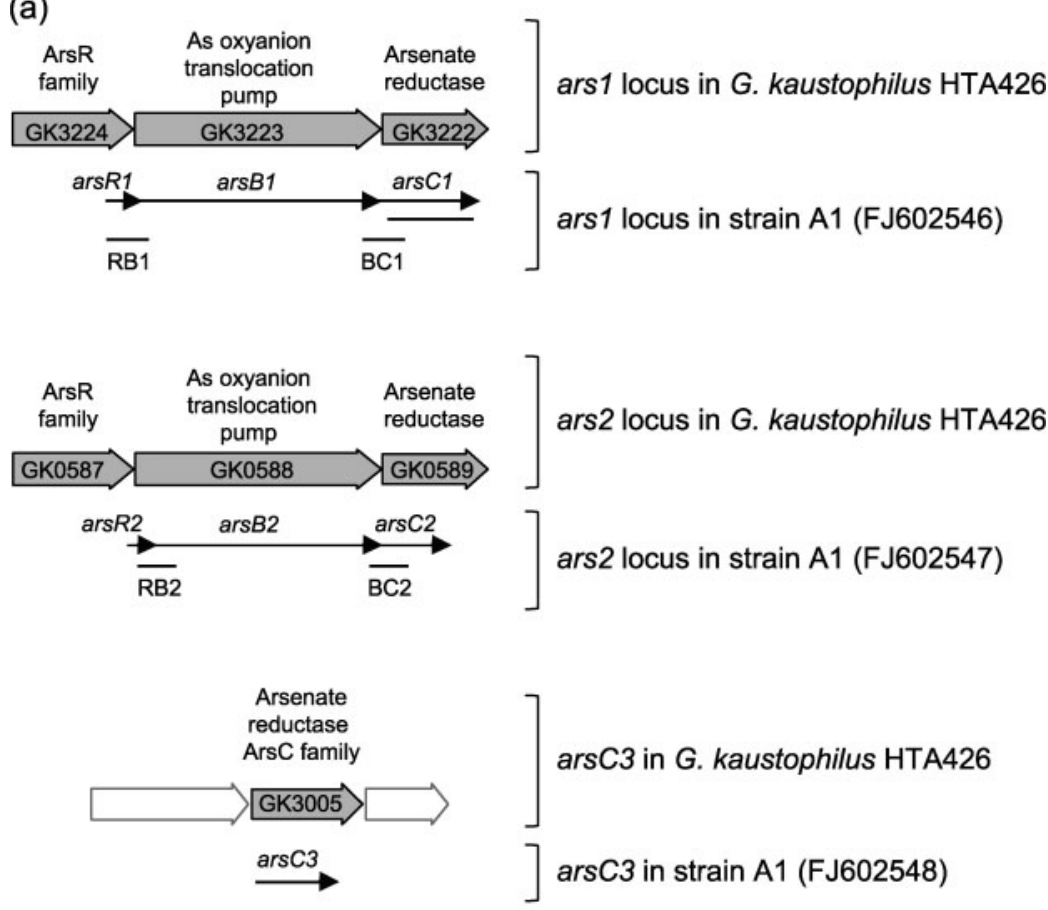

(b)

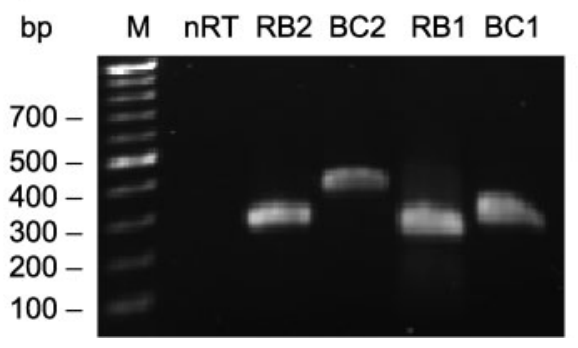

Fig. 2. Organization of arsenic-resistance genes in Geobacillus strains. (a) Three arsenic-resistance loci in G. kaustophilus HTA426 (redrawn from the genome annotation project) and the corresponding organization of the homologue operons derived for G. kaustophilus A1, projected from sequence data. Open arrows represent genes not involved in arsenic resistance. Segments correspond to sequenced regions that were assembled and deposited in GenBank, as indicated by the accession numbers in parentheses. (b) Co-transcription analysis relative to the ars 1 and ars2 operons. M, DNA markers; nRT, control without reverse transcriptase; RB2, BC2, RB1 and BC1, RT$\mathrm{PCR}$ products corresponding to the regions indicated in (a).

reductases belonging to this family require thioredoxin for arsenate reduction. The ArsC2 of $\mathrm{GK}$ is $85 \%$ identical to the arsenate reductase of Bacillus subtilis strain 168, an enzyme for which the structure has been solved and has been key to understanding the mechanisms of thioredoxin-arsenate reduction (Li et al., 2007; Bennett et al., 2001). In addition, the protein sequences of the two $\mathrm{As}(\mathrm{V})$ reductases $\mathrm{ArsCl}$ and ArsC2 show a high level of similarity to their homologues in the genomes of other Geobacillus species, including Geobacillus thermodenitrificans NG80-2, Geobacillus thermoglucosidasius C56-YS93, Geobacillus thermoleovorans, and Geobacillus sp. C56-T3, Y412MC52 and Y412MC10.

ORF GK3005, and the partial homologue sequence of strain A1, encode an arsenate reductase (ArsC3, 121 aa) which belongs to a second family of arsenate reductases, ArsC (Fig. 3). Sequences belonging to the ArsC family, first described in E. coli (Kaur \& Rosen, 1992; Silver et al., 1981), rely on reducing equivalents from glutathione (GSH) and are characterized by a thioredoxin (TRX) fold domain, a motif also found in thioredoxins and glutaredoxins. Because this family of reductases requires glutaredoxin to reduce arsenate, we used the GK genome as a guide and examined the region surrounding the ars operons and ars $\mathrm{C} 3$ in the GK annotation. Two genes encoding putative glutaredoxins were present in the genome, but in locations distant from the ars 3 locus. Instead, a thioredoxin gene was identified approximately $1.3 \mathrm{~kb}$ downstream of ars $\mathrm{C} 3$, although its presence might be unrelated to the function of ArsC3.

\section{Co-transcription of ars $R,-B$ and $-C$ in ars 1 and ars2}

To demonstrate that ars 1 and ars 2 constitute functional operons, their transcriptional analysis was carried out by RTPCR. Primers for the detection of specific ars $C$ transcripts, and for co-transcription analyses, were designed on the basis of the nucleotide sequences determined for Geobacillus strain A1 (Supplementary Table S1). RT-PCR analysis of the mRNA transcripts, using primers that amplified across contiguous genes, demonstrated that three genes, ars $R B C$, were co-transcribed in both operons, as indicated by segments $\mathrm{RB} 1, \mathrm{BC} 1, \mathrm{RB} 2$ and $\mathrm{BC} 2$ (Fig. 2b). The negative control nRT did not include the reverse transcriptase. 


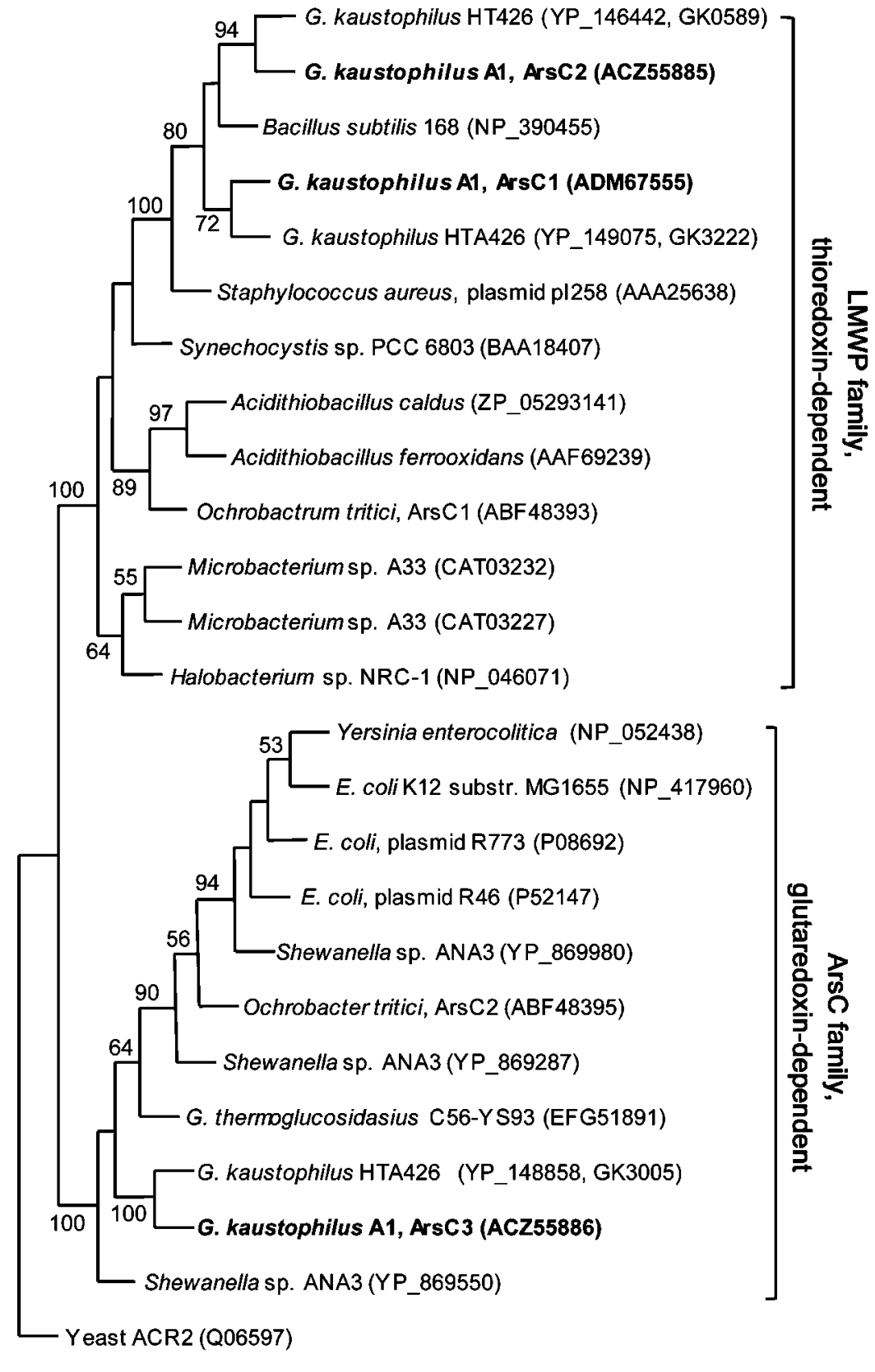

Fig. 3. Maximum-parsimony analysis based on 111 positions of arsenate reductase sequences. Protein accession numbers are in parentheses. Only bootstrap values greater than 50 are shown. The ACR2 yeast sequence was used as the outgroup.
Furthermore, the three reductases were transcribed in both the presence and the absence of arsenate (Fig. 4).

\section{Arsenate reductase genes are induced by arsenate}

Since transcripts corresponding to both the ars 1 operon and the ars 2 operon and to ars $\mathrm{C} 3$ were detectable, regardless of the presence of arsenate, to further investigate the role of the three putative ars $C$ genes the abundance of their transcripts was measured by qRT-PCR. Cells grown on LB to mid-exponential phase were treated with $5 \mathrm{mM}$ arsenate, and after 10 min total RNA was isolated. The abundance of each transcript, compared with untreated samples, is shown in Fig. 4. Expression of $\operatorname{ars} \mathrm{C} 1$ and $\operatorname{ars} \mathrm{C} 2$ was strongly induced in cells grown on arsenate, while the expression of arsC3 was minimally affected by arsenate. Furthermore, in the absence of arsenate, the abundance of the $\operatorname{ars} C 3$ transcript was slightly more elevated than that of $\operatorname{ars} C 1$ and $\operatorname{ars} C 2$. These results indicate that both $\operatorname{ars} C 1$ and ars $C 2$ are responsive to arsenate, presumably through the arsenite resulting from $\mathrm{As}(\mathrm{V})$ reduction after cell uptake.

\section{DISCUSSION}

A G. kaustophilus strain, designated A1 and isolated from a geothermal environment in Tuscany (Italy), was found to be highly resistant to arsenate, and also tolerated moderate 


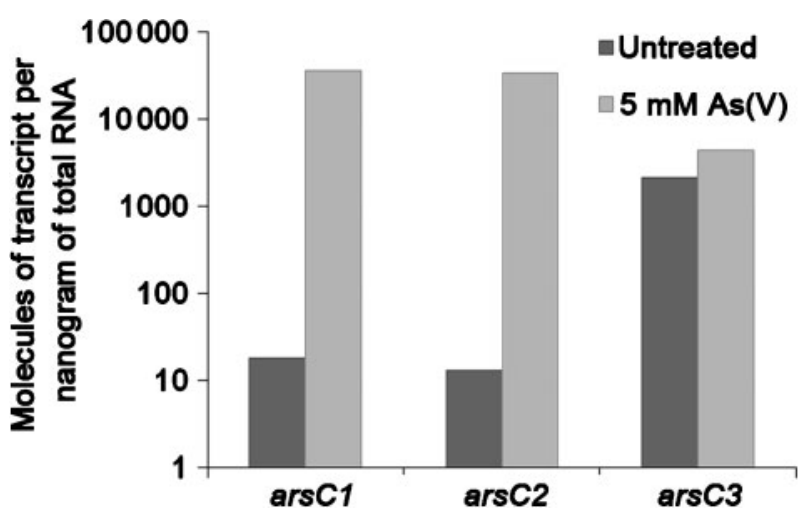

Fig. 4. Expression profile of the arsenate reductase coding genes ars $\mathrm{C} 1$, ars $\mathrm{C} 2$ and ars $\mathrm{C} 3$ in strain $\mathrm{A} 1$. Transcripts were quantified by real-time RT-PCR as molecules of transcript per nanogram of total RNA isolated from cultures grown with $5 \mathrm{mM}$ arsenate (arsC1, $36823 \pm 3933$; $\operatorname{arsC2}, 33883 \pm 4616$; $\operatorname{arsC3}, 4507 \pm 142)$ or without treatment (arsC1, 18 \pm 7 ; arsC2, 13 \pm 1 ; arsC3, $2126 \pm 200$ ). Results are reported as means of at least three experiments.

levels of arsenite and antimonite, and formed dark colonies in the presence of tellurate (Cuebas et al., 2011). In this report, we demonstrate that the resistance of strain A1 to arsenic is due to its ability to reduce $\mathrm{As}(\mathrm{V})$ to $\mathrm{As}(\mathrm{III})$, consistent with the presence of multiple genes encoding putative arsenate reductases and arsenite transporters, although additional mechanisms responsible for arsenic resistance cannot be excluded. The most common systems of arsenate resistance rely on the reduction of arsenate and the active efflux of the resulting arsenite (Rosen, 2002). In prokaryotes, arsenate reduction may be carried out for detoxification or for energy conservation, as an alternative method of anaerobic respiration (Oremland \& Stolz, 2003; Stolz et al., 2006). However, fundamentally different enzymes are responsible for these two modes of arsenate reduction (Messens \& Silver, 2006; Stolz et al., 2002). No growth was observed when Geobacillus strain A1 was incubated in the presence of $5 \mathrm{mM}$ arsenate as the sole terminal electron acceptor (Cuebas et al., 2011). This observation, together with the fact that no Arr sequences were identified in strain $\mathrm{A} 1$, indicates that this micro-organism does not respire arsenate. However, reduction of arsenate to arsenite was detected during aerobic growth (Fig. 1), suggesting that the transformation is mediated by a detoxifying arsenate reductase. To verify this hypothesis, specific PCR primers targeting known arsenic detoxification genes were designed on the basis of the chromosome sequence of GK. Such primers enabled the detection of one arsenate reductase of the ArsC family and two unlinked operons, each encoding a typical ars $R B C$ cluster, within strain A1.

The sequences of ars genes are highly conserved in the two strains A1 and GK, and their arrangement in strain A1 resembles very closely the organization of homologous genes in GK (Fig. 2). The genome sequences of several Geobacillus species are currently available, and from their inspection we observed that most of them (including G. thermodenitrificans NG80-2, G. thermoglucosidasius C56-YS93, G. stearothermophilus, and Geobacillus sp. Y412MC10, Y412MC61, C56-T3 and Y4.1MC1) possess a monocistronic reductase characterized by a TRX fold (ArsC family) and at least one arsRBC operon encoding an ArsC of the LMWP protein family (Supplementary Fig. S1). In contrast, Geobacillus sp. WCH70 encodes one arsenate reductase of each type, but none of them is part of an operon. GK and strain A1 appear unusual within their genus, because these strains possess two copies of the arsRBC operon (Fig. 2 and Supplementary Fig. S1). This characteristic is shared with B. subtilis (Supplementary Fig. S1), while other Bacillus species inspected at the J. Craig Venter Institute Comprehensive Microbial Resource (JCVI-CMR) database encode a single ars operon in their chromosome. It should be noted that the ars $C$ in one of the two ars operons of B. subtilis is interrupted by a frameshift. This may explain the difference at the level of arsenate tolerance between $G$. kaustophilus and B. subtilis ( 80 and $4 \mathrm{mM}$, respectively) (Cuebas et al., 2011; Sato \& Kobayashi, 1998) and the loss of arsenic resistance in Bacillus mutants lacking the ars operon of the skin element (Sato \& Kobayashi, 1998). It has been proposed that multiple ars operons contribute to elevated arsenate resistance (Li \& Krumholz, 2007), although it cannot be excluded that unusual arrangements of genes or their mode of regulation may also contribute to an increased resistance. The most arsenic-resistant species have been shown to rely on typical detoxification genes, chromosomally encoded but organized in unique ways: Microbacterium sp. strain A33 encodes an operon containing three arsenate reductases, one of which is fused to an arsRlike gene (Achour-Rokbani et al., 2010); Ochrobactrum tritici and Corynebacterium glutamicum have two structurally different chromosomal operons encoding arsenic-resistance genes (Branco et al., 2008; Ordóñez et al., 2005); and Desulfovibrio desulfuricans possesses an arsRBCC operon and one ars $C$ coding gene located in a separate chromosomal region (Li \& Krumholz, 2007). These bacteria display levels of arsenate resistance ranging from $50 \mathrm{mM}$ (D. desulfuricans) to $800 \mathrm{mM}$ (Microbacterium sp. A33). Except for Ochrobactrum, none of the other species mentioned possesses $\operatorname{ars} D$ or ars $A$ sequences. Nevertheless, other factors are likely to contribute to elevated arsenic resistance, as suggested by the observation that 'Ferroplasma acidarma$n u s^{\prime}$, an archaeon resistant to $>100 \mathrm{mM}$ arsenate, lacks arsenate reductase genes. Its chromosome encodes only an arsRB operon inducible by arsenite (Baker-Austin et al., 2007; Gihring et al., 2003). Other factors of resistance may involve strategies that render the cell membrane less permeable to arsenic compounds, such as downregulation of uptake systems, or yet to be identified genes that enhance the efficiency of the ars components.

Using RT-PCR we demonstrated that contiguous ORFs identified as putative arsenic-resistance genes in $G$. 
kaustophilus strain A1 were co-transcribed, constituting functional operons. Furthermore, the expression profiles of the three arsenate reductase coding genes, obtained by qRT-PCR, led us to develop a preliminary model for regulation of arsenic resistance in G. kaustophilus (Supplementary Fig. S2). Arsenate is easily imported by cells through phosphate transporters (Rosen, 2002). Given that the GK genome encodes the components of the phosphate-specific transport (Pst) system, we speculated that strain Al could take up As(V) through a similar system. The initial reduction of $\mathrm{As}(\mathrm{V})$ is probably carried out by $\mathrm{ArsC} 3$, the glutaredoxin-dependent reductase provided by the constitutive expression of arsC1. The As(III) that is generated during this reaction binds to both ArsR regulators, which are released from their respective promoters, thereby derepressing the ars 1 and ars 2 operons. The increased expression of the two arsenic-resistance operons results in the accumulation of transporters for As(III) efflux and an additional arsenate reductase, enabling the cells to tolerate high levels of arsenate in their surroundings. A similar model has been proposed for the arsenate response in $D$. desulfuricans (Li \& Krumholz, 2007), although the micro-organism has a single ars operon, and all of its three arsenate reductases belong to the LMWP family (Li \& Krumholz, 2007). The proposed model is to be considered a starting point to describe the elevated arsenic resistance of G. kaustophilus. Pending physiological questions include: what is the contribution of each ars gene to arsenic resistance, and what other factors, if any, might be responsible for the survival of the microbe in the presence of high arsenic levels? Furthermore, the fine details of the regulation of the ars genes remain the subject of further studies.

\section{ACKNOWLEDGEMENTS}

The work was supported by Hatch Project NJ01136 and a Pre-Tenure Award of the School of Environmental and Biological Sciences to E. B.

\section{REFERENCES}

Achour-Rokbani, A., Cordi, A., Poupin, P., Bauda, P. \& Billard, P. (2010). Characterization of the ars gene cluster from extremely arsenic-resistant Microbacterium sp. strain A33. Appl Environ Microbiol 76, 948-955.

Baker-Austin, C., Dopson, M., Wexler, M., Sawers, R. G., Stemmler, A., Rosen, B. P. \& Bond, P. L. (2007). Extreme arsenic resistance by the acidophilic archaeon 'Ferroplasma acidarmanus' Fer1. Extremophiles 11, 425-434.

Bennett, M. S., Guan, Z., Laurberg, M. \& Su, X. D. (2001). Bacillus subtilis arsenate reductase is structurally and functionally similar to low molecular weight protein tyrosine phosphatases. Proc Natl Acad Sci U S A 98, 13577-13582.

Branco, R., Chung, A. P. \& Morais, P. V. (2008). Sequencing and expression of two arsenic resistance operons with different functions in the highly arsenic-resistant strain Ochrobactrum tritici SCII24T. BMC Microbiol 8, 95.
Bruhn, D. F., Li, J., Silver, S., Roberto, F. \& Rosen, B. P. (1996). The arsenical resistance operon of IncN plasmid R46. FEMS Microbiol Lett 139, 149-153.

Cai, J. \& DuBow, M. S. (1996). Expression of the Escherichia coli chromosomal ars operon. Can J Microbiol 42, 662-671.

Chen, C. M., Misra, T. K., Silver, S. \& Rosen, B. P. (1986). Nucleotide sequence of the structural genes for an anion pump. The plasmidencoded arsenical resistance operon. J Biol Chem 261, 15030-15038.

Chini, V., Foka, A., Dimitracopoulos, G. \& Spiliopoulou, I. (2007). Absolute and relative real-time PCR in the quantification of tst gene expression among methicillin-resistant Staphylococcus aureus: evaluation by two mathematical models. Lett Appl Microbiol 45, 479484.

Cuebas, C., Sannino, D. \& Bini, E. (2011). Isolation and characterization of arsenic resistant Geobacillus kaustophilus strain from geothermal soils. J Basic Microbiol (Epub ahead of print).

Felsenstein, J. (2004). PHYLIP - Phylogeny Inference Package (Version 3.2). Cladistics 5, 164-166.

Fisher, J. C. \& Hollibaugh, J. T. (2008). Selenate-dependent anaerobic arsenite oxidation by a bacterium from Mono Lake, California. Appl Environ Microbiol 74, 2588-2594.

Gihring, T. M., Bond, P. L., Peters, S. C. \& Banfield, J. F. (2003). Arsenic resistance in the archaeon "Ferroplasma acidarmanus": new insights into the structure and evolution of the ars genes. Extremophiles 7, 123-130.

Jackson, M. D. \& Denu, J. M. (2001). Molecular reactions of protein phosphatases - insights from structure and chemistry. Chem Rev 101, 2313-2340.

Ji, G. \& Silver, S. (1992a). Reduction of arsenate to arsenite by the ArsC protein of the arsenic resistance operon of Staphylococcus aureus plasmid pI258. Proc Natl Acad Sci U S A 89, 9474-9478.

Ji, G. \& Silver, S. (1992b). Regulation and expression of the arsenic resistance operon from Staphylococcus aureus plasmid pI258. J Bacteriol 174, 3684-3694.

Kaur, P. \& Rosen, B. P. (1992). Plasmid-encoded resistance to arsenic and antimony. Plasmid 27, 29-40.

Larkin, M. A., Blackshields, G., Brown, N. P., Chenna, R., McGettigan, P. A., McWilliam, H., Valentin, F., Wallace, I. M., Wilm, A. \& other authors (2007). CLUSTAL W and CLUSTAL_X version 2.0. Bioinformatics 23, 2947-2948.

Li, X. \& Krumholz, L. R. (2007). Regulation of arsenate resistance in Desulfovibrio desulfuricans $\mathrm{G} 20$ by an arsRBCC operon and an $\operatorname{ars} C$ gene. J Bacteriol 189, 3705-3711.

Li, Y., Hu, Y., Zhang, X., Xu, H., Lescop, E., Xia, B. \& Jin, C. (2007). Conformational fluctuations coupled to the thiol-disulfide transfer between thioredoxin and arsenate reductase in Bacillus subtilis. J Biol Chem 282, 11078-11083.

Martin, P., DeMel, S., Shi, J., Gladysheva, T., Gatti, D. L., Rosen, B. P. \& Edwards, B. F. (2001). Insights into the structure, solvation, and mechanism of ArsC arsenate reductase, a novel arsenic detoxification enzyme. Structure 9, 1071-1081.

Mensink, E., van de Locht, A., Schattenberg, A., Linders, E., Schaap, N., Geurts van Kessel, A. \& De Witte, T. (1998). Quantitation of minimal residual disease in Philadelphia chromosome positive chronic myeloid leukaemia patients using real-time quantitative RT-PCR. Br J Haematol 102, 768-774.

Messens, J. \& Silver, S. (2006). Arsenate reduction: thiol cascade chemistry with convergent evolution. J Mol Biol 362, 1-17.

Mukhopadhyay, R. \& Rosen, B. P. (2002). Arsenate reductases in prokaryotes and eukaryotes. Environ Health Perspect 110 (Suppl. 5), $745-748$. 
Mukhopadhyay, R., Rosen, B. P., Phung, L. T. \& Silver, S. (2002). Microbial arsenic: from geocycles to genes and enzymes. FEMS Microbiol Rev 26, 311-325.

Murphy, J. N. \& Saltikov, C. W. (2009). The ArsR repressor mediates arsenite-dependent regulation of arsenate respiration and detoxification operons of Shewanella sp. strain ANA-3. J Bacteriol 191, 6722-6731.

Oden, K. L., Gladysheva, T. B. \& Rosen, B. P. (1994). Arsenate reduction mediated by the plasmid-encoded ArsC protein is coupled to glutathione. Mol Microbiol 12, 301-306.

Ordóñez, E., Letek, M., Valbuena, N., Gil, J. A. \& Mateos, L. M. (2005). Analysis of genes involved in arsenic resistance in Corynebacterium glutamicum ATCC 13032. Appl Environ Microbiol 71, 6206-6215.

Oremland, R. S. \& Stolz, J. F. (2003). The ecology of arsenic. Science 300, 939-944.

Pérez-Jiménez, J. R., DeFraia, C. \& Young, L. Y. (2005). Arsenate respiratory reductase gene ( $\operatorname{arr} A)$ for Desulfosporosinus sp. strain Y5. Biochem Biophys Res Commun 338, 825-829.

Rosen, B. P. (1999). Families of arsenic transporters. Trends Microbiol 7, 207-212.

Rosen, B. P. (2002). Biochemistry of arsenic detoxification. FEBS Lett 529, 86-92.

Rosen, B. P., Dey, S., Dou, D., Ji, G., Kaur, P., Ksenzenko M. Yu., Silver, S. \& Wu, J. (1992). Evolution of an ion-translocating ATPase. Ann N Y Acad Sci 671, 257-272.

Rosenstein, R., Peschel, A., Wieland, B. \& Götz, F. (1992). Expression and regulation of the antimonite, arsenite, and arsenate resistance operon of Staphylococcus xylosus plasmid pSX267. J Bacteriol 174, 3676-3683.

Sambrook, J., Fritsch, E. F. \& Maniatis, T. (1989). Molecular Cloning: a Laboratory Manual, 2nd edn. Cold Spring Harbor, NY: Cold Spring Harbor Laboratory.

Santini, J. M., Streimann, I. C. \& van den Hoven, R. N. (2004). Bacillus macyae sp. nov., an arsenate-respiring bacterium isolated from an Australian gold mine. Int J Syst Evol Microbiol 54, 2241-2244.

Sato, T. \& Kobayashi, Y. (1998). The ars operon in the skin element of Bacillus subtilis confers resistance to arsenate and arsenite. J Bacteriol 180, 1655-1661.

Shivaji, S., Suresh, K., Chaturvedi, P., Dube, S. \& Sengupta, S. (2005). Bacillus arsenicus sp. nov., an arsenic-resistant bacterium isolated from a siderite concretion in West Bengal, India. Int J Syst Evol Microbiol 55, 1123-1127.

Silver, S., Budd, K., Leahy, K. M., Shaw, W. V., Hammond, D., Novick, R. P., Willsky, G. R., Malamy, M. H. \& Rosenberg, H. (1981). Inducible plasmid-determined resistance to arsenate, arsenite, and antimony (III) in Escherichia coli and Staphylococcus aureus. J Bacteriol 146, 983996.

Silver, S., Ji, G., Bröer, S., Dey, S., Dou, D. \& Rosen, B. P. (1993). Orphan enzyme or patriarch of a new tribe: the arsenic resistance ATPase of bacterial plasmids. Mol Microbiol 8, 637-642.

Stauffer, R. E. \& Thompson, J. M. (1984). Arsenic and antimony in geothermal waters of Yellowstone National Park, Wyoming, USA. Geochim Cosmochim Acta 48, 2547-2561.

Stolz, J. F., Basu, P. \& Oremland, R. S. (2002). Microbial transformation of elements: the case of arsenic and selenium. Int Microbiol 5, 201-207.

Stolz, J. F., Basu, P., Santini, J. M. \& Oremland, R. S. (2006). Arsenic and selenium in microbial metabolism. Annu Rev Microbiol 60, 107130.

Switzer Blum, J., Burns Bindi, A., Buzzelli, J., Stolz, J. F. \& Oremland, R. S. (1998). Bacillus arsenicoselenatis, sp. nov., and Bacillus selenitireducens, sp. nov.: two haloalkaliphiles from Mono Lake, California that respire oxyanions of selenium and arsenic. Arch Microbiol 171, 19-30.

Takami, H., Takaki, Y., Chee, G. J., Nishi, S., Shimamura, S., Suzuki, H., Matsui, S. \& Uchiyama, I. (2004). Thermoadaptation trait revealed by the genome sequence of thermophilic Geobacillus kaustophilus. Nucleic Acids Res 32, 6292-6303.

Wang, G., Kennedy, S. P., Fasiludeen, S., Rensing, C. \& DasSarma, S. (2004). Arsenic resistance in Halobacterium sp. strain NRC-1 examined by using an improved gene knockout system. J Bacteriol 186, 31873194.

Xu, C. \& Rosen, B. P. (1997). Dimerization is essential for DNA binding and repression by the ArsR metalloregulatory protein of Escherichia coli. J Biol Chem 272, 15734-15738.

Zargar, K., Hoeft, S., Oremland, R. \& Saltikov, C. W. (2010). Identification of a novel arsenite oxidase gene, $\operatorname{arxA}$, in the haloalkaliphilic, arsenite-oxidizing bacterium Alkalilimnicola ehrlichii strain MLHE-1. J Bacteriol 192, 3755-3762.

Edited by: E. L. Madsen 